\title{
About the series
}

This book aims to use the applications of Computational Intelligence and Predictive Analysis for medical science in the rapidly changing world and benefit society in doing research, innovation and development in this field. For fostering research in this high-potential emerging area, this book is relevant to a variety of data that contribute to the challenges faced by medical science. Technology has caused a complete shift in the way people experience the world and how they live their lives. The world is now a global village, and technology has caught the world by advancements that are being made with each passing day are without doubt hitting the world with a bang. This new-age technology is ready to aid us through the disciplines of Computational Intelligence and Predictive Analysis for medical science. 
